\title{
Acknowledgment to JCMK Editorial Board and Peer-Reviewers for contribution in 2013-2020
}

\section{Laura Dybyssova}

Executive Secretary, on behalf of editorial team of Journal of Clinical Medicine of Kazakhstan

On behalf of Journal of Clinical Medicine of Kazakhstan, we would like to express our appreciation to all editorial and advisory board members, reviewers and authors who contributed to this journal for 2013-2020 years.

Beginning from 2021, the Journal of Clinical Medicine of Kazakhstan transferred to new editorial submission system (EditorialPark). The members of Editorial and Advisory board have been updated.

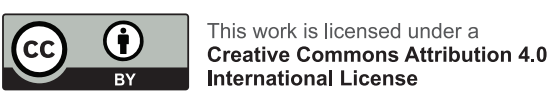

\section{EDITORIAL AND ADVISORY BOARD MEMBERS IN 2013-2020}

\section{Editor-In-Chief}

Abay Baigenzhin (Kazakhstan)

\section{Editors}

Temirlan Karibekov (Kazakhstan)

Saltanat Tuganbekova (Kazakhstan)

\section{Associate Editor}

Abduzhappar Gaipov (Kazakhstan)

\section{Executive Secretary}

Laura Dybyssova (Kazakhstan)

\section{Editorial Board}

Manarbek Askarov (Kazakhstan)

Zulfiya Orynbayeva (USA)

Rimantas Benetis (Lithuania)

Galina Fedotovskikh (Kazakhstan)

Ospan Mynbaev (Russian Federation)

Gunay Akbarova (Azerbaijan)

Selman Unverdi (Turkey)

Ulan Kabayev (Kazakhstan)

Talgat Nurgozhin (Kazakhstan)

Yuriy Kazakov (Ukraine)

Almaz Makenjan uulu (Kyrgyz Republic)

Jakhongir Alidjanov (Uzbekistan)

Praveen Kumar Potukuchi (USA)

Oybek Rustamov (Australia)

Rustam Mustafin (Bashkortostan,

Russian Federation)

Adem Kucuk (Turkey)

Yana Sotskaya (Ukraine)
Ainura Dzhangaziyeva (Kyrgyz Republic)

Mehtap Tinazli (Turkey)

Yulia Lunitsyna (Russian Federation)

Yuksel Ersoy (Turkey)

Rikhsi Sabirova (Uzbekistan)

Nurdin Mamanov (Kyrgyz Republic) Mariya Derbak (Ukraine)

National Advisory Board

Suyundik Imankulov (Kazakhstan)

Anatoliy Kolos (Kazakhstan)

Vitaliy Koikov (Kazakhstan)

Saule Abseitova (Kazakhstan)

Almagul Kushugulova (Kazakhstan)

Marlen Doskali (Kazakhstan)

Kakharman Yesmembetov (Kazakhstan)

Nelya Bissenova (Kazakhstan)

Gauri Bapayeva (Kazakhstan)

Ibragim Tashev (Kazakhstan)

Bagdat Imasheva (Kazakhstan)

Galiya Shaimardanova (Kazakhstan)

Nasrulla Shanazarov (Kazakhstan)

Adilzhan Albazarov (Kazakhstan)

Elmira Chuvakova (Kazakhstan)

International Advisory Board Turgut Teke (Turkey)

Kubes Jiri (Czech Republic)

Yaroslav Tolstyak (Ukraine)

Zhannat Taubaldiyeva (Kazakhstan)

Aidos Konkayev (Kazakhstan)

Samat Saparbayev (Kazakhstan)

Olga Ulyanova (Kazakhstan)

Galiya Orazova (Kazakhstan) 


\section{PEER-REVIEWERS WHO CONTRIBUTED TO THIS JOURNAL}

\section{DURING 2013-2020}

Abay K. Baigenzhin (Kazakhstan)

Abdulkadir Basturk (Turkey)

Abdurashid Mussayev (Kazakhstan)

Abduzhappar Gaipov (Kazakhstan)

Ahmed Alshewered (Iraq)

Ahmet Taylan Çebi (Turkey)

Aigerim Mussina (Kazakhstan)

Aigul Abduldayeva (Kazakhstan)

Aigul Abduldayeva (Kazakhstan)

Aigul Dinmukhamedova (Kazakhstan)

Ainura Dzhangaziyeva (Kyrgyzstan)

Aiymkul Ashimkhanova (Kazakhstan)

Akbar Mahmood (Oman)

Akerke Jarbolova (Kazakhstan)

Akinsola Aina (Nigeria)

Akmaral Kassymova (Kazakhstan)

Aleksey Pak (Kazakhstan)

Alexander Kutsyn (Ukraine)

Ali Şahin (Turkey)

Alireza Rezai (Iran)

Aliya Sadykova (Kazakhstan)

Aliya Dzholdasbekova (Kazakhstan)

Aliya Tokusheva (Kazakhstan)

Aliya Seidullayeva (Kazakhstan)

Alma Shamova (Kazakhstan)

Almagul Kushugulova (Kazakhstan)

Almas Kussainov (Kazakhstan)

Almaz Makenzhan Uulu (Kyrgyzstan)

Alpamys Issanov (Kazakhstan)

Amangali Akanov (Kazakhstan)

Anara Abbay (Kazakhstan)

Anargul Nogayeva (Kazakhstan)

Andrey Semonenko (Ukraine)

Ankur Dashputre (United States)

Anna Krylova (Ukraine)

Anna Lushan (Ukraine)

Ardak Ainakulov (Kazakhstan)

Arsheed Bhat (India)

Arystan Seydalin (Kazakhstan)

Ashish Jaiman (India)

Askhat Myngbay Myngbay (Kazakhstan)

Assem Makhatova (Kazakhstan)

Assiya Turgambayeva (Kazakhstan)

Athanasios Zafeirakis (Greece)

Ayca Nazli Bulut (Turkey)

Ayk Mirzoyan (Armenia)

Ayla Kanbur (Turkey)

Aylin Aydın Sayılan (Turkey)

Ayten Yilmaz (Turkey)

Bagdat Ashimbekova (Kazakhstan)

Baglan Mustafayev (Kazakhstan)

Bakhytgul Yermekbayeva (Kazakhstan)

Bakhytzhan Zhunissov (Kazakhstan)

Bakytzhan Bimbetov (Kazakhstan)

Beglan Stambol (Kazakhstan)

Berik Tuleubayev (Kazakhstan)

Berrin Inanc (Turkey)

Betül Sargın (Turkey)

Bircan Yucekaya (Turkey)

Bogdan Kondratskiy (Ukraine)

Bolat Saktashev (Kazakhstan)

Bora Bilal (Turkey)

Boris Larionov (Russia)

Bota Kuanova (Kazakhstan)
Burhan Fatih Koçyiğit (Turkey)

Calin Pop (Romania)

Capros Capros (R Moldova)

Chee Kai Chan (Kazakhstan)

Cihangir Doğu (Turkey)

Damirbek Osmonov (Kazakhstan)

Dias Yeleshov (Kazakhstan)

Didem Baskin Embleton (Turkey)

Dinara Galiyeva (Kazakhstan)

Dinara Jumadilova (Kazakhstan)

Dmitriy Malykh (Germany)

Dmitriy Viderman (Kazakhtan)

Ensar Durmus (Turkey)

Ergun Bozoglu (Turkey)

Erkan Karacan (Turkey)

Erol Demir (Turkey)

Ertan Altaylı (Turkey)

Esra Polat (Turkey)

Ethem Bilgiç (Turkey)

Fakhriddin Sarzhanov (Turkey)

Fatih Tezcan (Turkey)

Fatih Tanrıverdi (Turkey)

Fatma Kulali (Turkey)

Fatma Kızılay (Turkey)

Fatma Yilmaz Karadağ (Turkey)

Faye Foster (United Kingdom)

Fevzi Cengiz (Turkey)

Filiz Özdemir (Turkey)

Fulya Bakılan (Turkey)

Funda Levendoglu (Turkey)

Galina Koval (Ukraine)

Galina Fedatovskikh (Kazakhstan)

Galiya Orazova (Kazakhstan)

Galiya Abdulina (Kazakhstan)

Gaukhar Bakhtiyarova (Kazakhstan)

Gauri Bapayeva (Kazakhstan)

Genco Gencdal (Turkey)

Gökhan Özdemir (Turkey)

Görker Sel (Turkey)

Grace Cornelius (India)

Gulbaram Ustenova (Kazakhstan)

Gülçin Gazioğlu Türkyılmaz (Turkey)

Gulmira Janalina (Kazakhstan)

Gulnar Rakhimbekova (Kazakhstan)

Gulnar Taitubayeva (Kazakhstan)

Gulnar Zhaksylykova (Kazakhstan)

Gulnara Bedelbayeva (Kazakhstan)

Gulnara Gurtskaya (Kazakhstan)

Gulsara Imambayeva (Kazakhstan)

Gulshat Atabayeva (Kazakhstan)

Gulshat Kemelova (Kazakhstan)

Gulzhan Assemova (Kazakhstan)

Gulzhan Omurkulova (Kyrgyzstan)

Gulzhanat Aimagambetova (Kazakhstan)

Gulziya Ismailova (Kazakhstan)

Gunay Akbarova (Azerbaijan)

Gurpreet Singh (Malaysia)

Habib Emre (Turkey)

Hasan Khalaf (United Kingdom)

Hasan Bingöl (Turkey)

Hasret Ayyildiz Civan (Turkey)

Hayriye Şahinli (Turkey)

Hilal Erken Pamukcu (Turkey)

Hülya Nazik (Turkey)
Huseyin Solgun (Turkey)

Hüseyin Serinçay (Turkey)

Ibrahim Inanc (Turkey)

Igor Samoilenko (Ukraine)

ljeoma Obumneme-Anyim (Nigeria)

İlknur Albayrak (Turkey)

İlknur Ucuz (Turkey)

İnci Fatma Dogan (Turkey)

Inkar Mansurova (Kazakhstan)

Irina Krivulya (Ukraine)

Irina Teryshina (Ukraine)

Irina Kuzmina (Ukraine)

Islam Madadov (Kazakhstan)

Ivan Mironenko (Ukraine)

Ivana Dewi (Indonesia)

Izuchukwu Okafor (Nigeria)

Jakhongir Alidjanov (Uzbekistan)

Joseph Almazan (Philippines)

Kainar Kadyrzhanuly (Kazakhstan)

Kakharman Yesmembetov (Kazakhstan)

Kaldanay Kozhanova (Kazakhstan)

Karlygash Kuzembayeva (Kazakhstan)

Konstantin Garkalov (Kazakhstan)

Kristina Chimpoi (Ukraine)

Kseniya Shugareva (Kazakhstan)

Kuanysh Yergaliyev (Kazakhstan)

Kuat Oshakbayev (Kazakhstan)

Kulsara Rustemova (Kazakhstan)

Kuralay Atageldiyeva (Kazakhstan)

Laura Dybyssova (Kazakhstan)

Leila Zholymbekova (Kazakhstan)

Leila Appazova (Kazakhstan)

Liliya Omarova (Kazakhstan)

Mahdi Zaid (Palestine)

Mahir Tayfur (Turkey)

Maira Yespenbetova (Kazakhstan)

Maksim Potjagenko (Ukraine)

Manshuk Eltokova (Kazakhstan)

Maral Nogayeva (Kazakhstan)

Marat Gallyamov (Australia)

Marina Khvan (Kazakhstan)

Mariya Derbak (Ukraine)

Mariya Rybitskaya (Ukraine)

Mariya Prilutskaya (Kazakhstan)

Marzhan Rakhimzhanova (Kazakhstan)

Mehmet Türkeli (Turkey)

Mehmet Bankir (Turkey)

Mehmet Ali Kobat (Turkey)

Mehtap Tinazli (Turkey)

Mohamad Aljofan (Australia)

Mohamed Elrggal (Egypt)

Muhammad Khan (Pakistan)

Mujeeb ur Rahman (China)

Mukhit Dossov (Kazakhstan)

Munira Karimova (Uzbekistan)

Mustafa Bilgi (Turkey)

Mustafa Atalay (Turkey)

Nailya Toktarova (Kazakhstan)

Nataliya Chekalina (Ukraine)

Natalya Abramova (Ukraine)

Natalya Glushkova (Kazakhstan)

Nelya Bissenova (Kazakhstan)

Nevin Karakuş (Turkey)

Nurdin Mamanov (Kyrgyzstan) 
Odil Kamalkhanovich (Uzbekistan) Oğuzhan Arun (Turkey) Oksana Feshchuk (Ukraine) Oksana Drapkina (Russia) Olayinka Ibrahim (Katsina) Oleg Tsoy (Kazakhstan) Oleg Gaisenok (Russia) Olga Ulyanova (Kazakhstan) Olga Ignatenko (Kazakhstan) Ospan Mynbaev (Russia) Oybek Rustamov (Australia) Özgür Yağan (Turkey) Pakhitkanym Ishuova (Kazakhstan) Paolo Colet (Kazakhstan)

Petar Avramovski (Macedonia) Praveen Potukuchi (United States) Rahim Tojiboyev (Kazakhstan) Rakhima Mekenbayeva (Kazakhstan) Raushan Alibekova (Kazakhstan) Reha Girgin (Turkey) Reza Rezaie Khanghah (Iran) Rikhsi Sabirova (Uzbekistan) Rizaldy Pinzon (Indonesia) Rodion Konovalov (Kazakhstan) Rohat Ak (Turkey)

Roin Rekvava (Kazakhstan) Rosa Karabekova (Kazakhstan) Rustam Mustafin (Russia) Safiye Sanem Dereli Bulut (Turkey) Sakhiba Yakubbekova (Uzbekistan) Saliya Bissimbayeva (Kazakhstan) Saltanat Dzhetybayeva (Kazakhstan) Samal Sarsenova (Kazakhstan) Samat Kozhakhmetov (Kazakhstan) Saule Abseitova (Kazakhstan) Saule Kubekova (Kazakhstan) Selcuk Gulmez (Turkey)
Selçuk Nazik (Turkey)

Selin Gaş (Turkey)

Selman Unverdi (Turkey)

Sergey Shandyba (Ukraine)

Sergey Shirinkin (Ukraine)

Serik Nurmaganov (Kazakhstan)

Sezgin Tekin (Turkey)

Sezgin Gunes (Turkey)

Shah Murad (Pakistan)

Shalkar Adambekov (Kazakhstan)

Sholpan Suleymenova (Kazakhstan)

Sholpan Nurmanova (Kazakhstan)

Sholpan Kulzhanova (Kazakhstan)

Sinan Kardes (Turkey)

Sohul Shuvo (United States)

Sonay Goktas (Turkey)

Songul Cuglan (Turkey)

Suantak Vaiphei (India)

Suindyk Imankulov (Kazakhstan)

Suriya Yessentayeva (Kazakhstan)

Susana Jerez (Argentina)

Tae Bumsik (Republic of Korea)

Tatiana Melikova (Ukraine)

Tatyana Ponomareva (Kazakhstan)

Tatyana Burkutbayeva (Kazakhstan)

Timur Sarsengaliyev (Kazakhstan)

Tuba Tülay Koca (Turkey)

Tülin Yildiz (Turkey)

Turan Yildiz (Turkey)

Turgut Teke (Turkey)

Ulan Zhaparov (Kazakhstan)

Ulanbek Balpukov (Kazakhstan)

Uldan Derbisbekova (Kazakhstan)

Ulugbek Mazhidov (Turkey)

Umida Zakirova (Uzbekistan)

Valentina Delevskaya (Ukraine)

Valentina Stolyarova (Kazakhstan)
Vasiliki Holeva (Greece)

Venera Altynova (Kazakhstan)

Venera Omarova (Kazakhstan)

Vitaliy Koikov (Kazakhstan)

Vitaliy Sazonov (Kazakhstan)

Vladimir Pak (Kyrgyzstan)

Yana Yugan (Ukraine)

Yana Sotskaya (Ukraine)

Yannick Nlandu (Democratic Republic of

Congo)

Yaroslav Tolsyak (Ukraine)

Yasin Uzuntarla (Turkey)

Yelena Khmel (Ukraine)

Yelena Zholdybayeva (Kazakhstan)

Yerbolat Dalenov (Kazakhstan)

Yodang Yodang (Indonesia)

Yousef Mortazavi (Iran)

Yüksel Ersoy (Turkey)

Yuliya Lunitsyna (Russia)

Yuriy Blikharski (Ukraine)

Yuriy Kolossov (Kazakhstan)

Yuriy Olkhovik (Kazakhstan)

Yuriy Kobeliatsky (Ukraine)

Zahraa Motaweq (Iraq)

Zhanar Yermakhanova (Kazakhstan)

Zhandos Amankulov (Kazakhstan)

Zhandulla Nakipov (Kazakhstan)

Zhannat Sadyrova (Kazakhstan)

Zhannat Taubaldiyeva (Kazakhstan)

Zhannat Mussabekova (Kazakhstan)

Zhansulu Akylzhanova (Kazakhstan)

Zholtay Daribayev (Kazakhstan)

Zia Choudhry (United States)

Zufar Abdullayev (Uzbekistan)

Zulfiya Orynbayeva (United States) 